\title{
Histological Appearance of Placental Solomonization in the Treatment of Twin-Twin Transfusion Syndrome
}

\author{
Stephen P. Emery, MD ${ }^{1,2}$ Lananh Nguyen, MD $^{3}$ \\ ${ }^{1}$ Department of Obstetrics, Gynecology and Reproductive Sciences, \\ School of Medicine, University of Pittsburgh, Pittsburgh, \\ Pennsylvania \\ 2 The Magee-Womens Research Institute, Pittsburgh, Pennsylvania \\ ${ }^{3}$ Department of Pathology, School of Medicine, University of \\ Pittsburgh, Pittsburgh, Pennsylvania
}

W. Tony Parks, $\mathrm{MD}^{1,2,3}$

Am J Perinatol Rep 2016;6:e165-e169.

\author{
Address for correspondence Stephen P. Emery, MD, Department of \\ Obstetrics, Gynecology and Reproductive Sciences, School of \\ Medicine, University of Pittsburgh, 300 Halket Street, Pittsburgh, PA \\ 15213 (e-mail: semery@mail.magee.edu).
}

\begin{abstract}
Keywords

- laser photocoagulation

- placental equatorialization

- Solomon technique

- twin-twin transfusion syndrome

Background Placental laser equatorialization or "solomonization" during treatment for twin-twin transfusion syndrome (TTTS) is associated with improved pregnancy outcomes. Concern exists, however, about the extent of placental injury caused by the technique, and of its ability to create "dichorionization," or complete separation of the fetal vascular circuits.

Case Study A "solomonized" placenta was histologically examined for depth of cell damage. Solomonization produces complete devascularization of the chorionic plate, but has minimal effect on the underlying villi. The median depth of the effect was $2,823 \mu \mathrm{m}$, or $<3 \mathrm{~mm}$.

Conclusion Solomonization produces complete devascularization of the chorionic plate, but has little effect on the underlying villi. "Dichorionization" is therefore not achieved. Ongoing surveillance of treated pregnancies is warranted.
\end{abstract}

Twin-twin transfusion syndrome (TTTS) is a serious complication of monochorionic (MC) twin pregnancies, affecting approximately $10 \%$ of MC gestations. It accounts for the majority of morbidity and mortality in monochorionic gestations. ${ }^{1}$ Fetoscopic laser photocoagulation has been demonstrated to improve pregnancy outcomes by cauterizing intertwin vascular anastomoses, the likely source of intertwin volume and vasoactive element exchange, and is considered to be the standard of care for the treatment of severe TTTS. ${ }^{2}$ Improvements in surgical technique (selective ablation, sequential selective ablation) have led to incremental but significant improvements in pregnancy outcomes, such that many centers report dual survival rates of 60 to $70 \%{ }^{3}$ Recurrent TTTS and twin anemia polycythemia sequence (TAPS) complicate approximately 14 and $13 \%$ of the treated cases, respectively. ${ }^{4}$ These conditions are felt to be due to incomplete treatment of the vascular anastomoses (incorrectly classified as paired, missed, or revascularized after treatment), and subsequent ongoing intertwin transfusion. ${ }^{5}$ Their treatment is more complicated than primary midtrimester TTTS for a myriad of technical reasons (presence of fluid in the donor's sac, membrane separation, preterm premature rupture of membranes, etc.). Therefore, a technique that reduces these outcomes would represent progress.

The most recent modification to fetoscopic laser coagulation is the "Solomon technique," or "solomonization," whereby a coagulation line is drawn by the laser along the entire vascular equator, connecting the individual cauterization points, in an effort to treat as many nonvisualized placental anastomoses as possible. Solomonization has been demonstrated to decrease the number of residual anastomoses by placental injection studies as well as decrease the likelihood of recurrent TTTS or of TAPS in a received

January 15, 2016 accepted after revision March 3, 2016
DOI http://dx.doi.org/

10.1055/s-0036-1582449. ISSN 2157-6998.
Copyright (c) 2016 by Thieme Medical Publishers, Inc., 333 Seventh Avenue, New York, NY 10001, USA. Tel: +1(212) 584-4662.
License terms

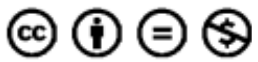


randomized controlled trial. ${ }^{6,7}$ Questions remain, however, about the depth of placental injury caused by the Solomon technique, and the completeness of "dichorionization," or complete separation of the two vascular circuits. The purpose of this study is to describe the histological appearance of the placenta following solomonization.

\section{Case Study}

The placenta from a 32-year-old Gravida 4, para 1111 (prior preterm birth at 22 weeks) who underwent sequential selective laser photocoagulation followed by solomonization at 20 weeks 5 days for the diagnosis of stage II TTTS was submitted for pathological analysis after delivery. Fifteen anastomoses were identified and cauterized in a sequential selective fashion using a $600 \mu \mathrm{m}$ diode laser (Dornier Medilas, Dornier MedTech America, Inc., Kennesaw, GA) at a power setting of $60 \mathrm{~W}$. Solomonization was performed at a power setting of $40 \mathrm{~W}$ : the individual cauterized anastomosis sites were connected by a single continuous cauterization line along the vascular equator and extended to the placental edges. Postoperative ultrasound surveillance documented normalization of fluid volumes with no evidence of injury to the intertwin membrane. There was no evidence of recurrent TTTS and no evidence of TAPS on middle cerebral artery Doppler surveillance. Both twins were live born after preterm labor at 25 weeks. Baby A, the former donor, weighed $677 \mathrm{~g}$ with Apgar scores of 4 and 7, at 1 and 5 minutes, respectively. Hemoglobin and hematocrit values on complete blood cell count for baby A were $12.2 \mathrm{mg} / \mathrm{dL}$ and 34.9\%, respectively. Baby $\mathrm{B}$, the former recipient, weighed $720 \mathrm{~g}$ with Apgar scores of 4 and 7 , at 1 and 5 minutes, respectively. Hemoglobin and hematocrit values were $14.6 \mathrm{mg} / \mathrm{dL}$ and $42.4 \%$, respectively.
The placenta was submitted for pathological analysis after delivery. The line of solomonization was identified along the surface of the fresh specimen. A total of 18 sequential full-thickness sections of the placenta along the entire line of solomonization (including several treated anastomotic sites) were formalin-collected, fixed, paraffinembedded, and hematoxylin-eosin ( $H \& E$ ) stained for microscopic evaluation. The University of Pittsburgh Institutional Review Board approved this study.

Macroscopic examination demonstrated a 199-g monochorionic diamniotic twin placenta with one centrally inserted and one marginally inserted three-vessel umbilical cord. A tan, irregular $17 \times 1 \mathrm{~cm}$ line on the placental disc was present indicative of the line of solomonization ( $\mathbf{- F i g . 1} \mathbf{1}$ ).

-Fig. 2 demonstrates the microscopic effects of solomonization along the vascular equator of the placenta at low magnification. -Fig. 3 shows the homogeneous eosinophilic region corresponding to complete devitalization of the chorionic plate with complete loss of nuclei in the amnion and chorion and underlying superficial villi. As one progresses toward the maternal surface, - Fig. 4 shows an abrupt transition between the avascular and vascular regions within the villi juxtaposed to the chorionic plate, which represents the depth of solomonization. - Fig. 5 compares an untreated to a treated section of placenta and demonstrates that the deeper villi are intact and essentially unaffected by the solomonization procedure.

The placental lesions were quantitated on $\mathrm{H} \& \mathrm{E}$ sections using the SPOT imaging software (SPOT Imaging, Sterling Heights, MI) (available at: www.spotimaging.com) to assess the relative area of placenta solomonized in the vascular equator. The shallowest depth of placental penetration by solomonization was $1,029 \mu \mathrm{m}$ whereas the deepest was 4,174

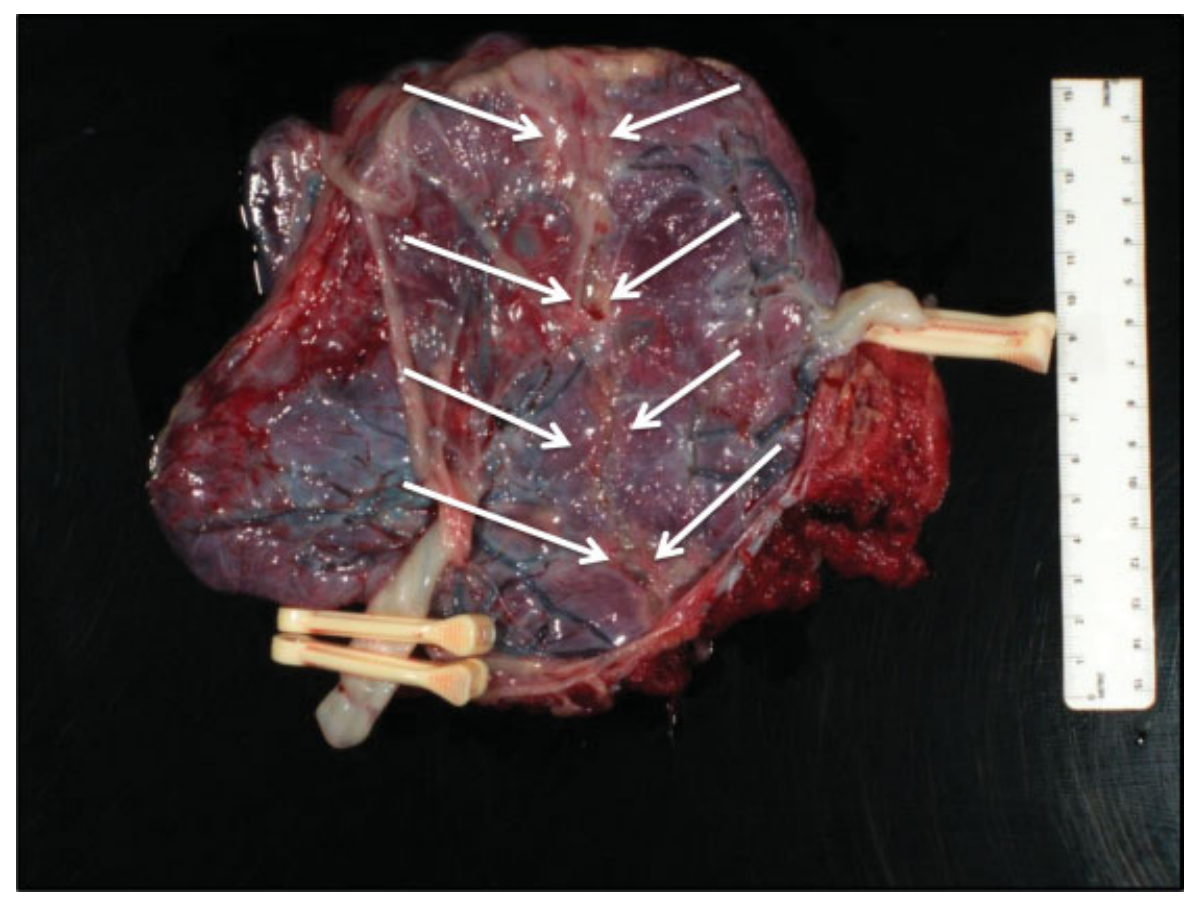

Fig. 1 Gross image of the placenta. White arrows denote the line of solomonization. 


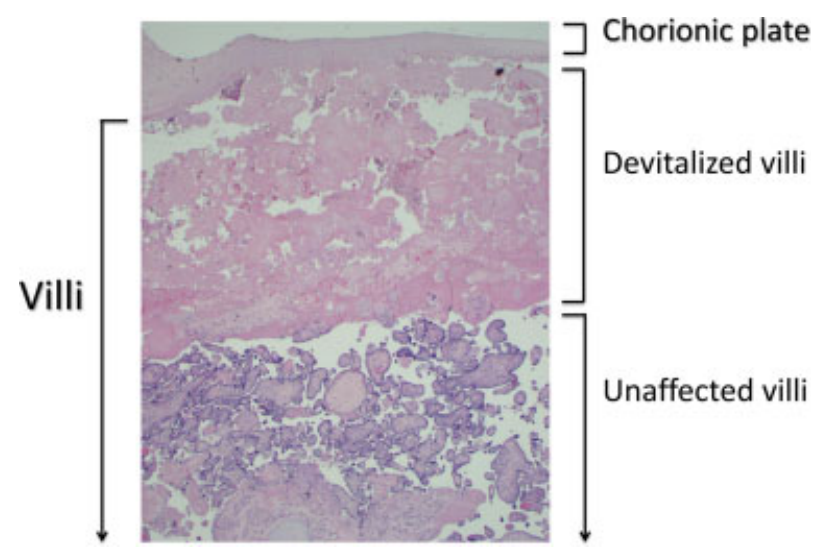

Fig. 2 Low-power magnification of an H\&E-stained section of the solomonization line. H\&E, hematoxylin-eosin.

$\mu \mathrm{m}$. The average depth affected was $2,597 \mu \mathrm{m}$ ( - Table $\mathbf{1})$. This is in contrast to the depth of penetration of a treated anastomosis, which was $1,558 \mu \mathrm{m}$ (-Fig. 6). The average thickness of the chorionic plate was $307 \mu \mathrm{m}$.

\section{Discussion}

TAPS and recurrent TTTS are significant complications of fetoscopic laser coagulation, occurring at 13 to $16 \%$ and 7 to $14 \%$ of cases, respectively. ${ }^{4,6}$ Management options, especially for TAPS, are less straightforward than for primary TTTS, and include repeat fetoscopic laser coagulation, intrauterine transfusion, selective feticide, pregnancy termination, and preterm delivery. Therefore, any technical innovation that would decrease the rate of these complications would be welcome, as dual survival remains the ultimate, yet still elusive, goal. Solomonization has been shown to decrease the rate of these complications, as well as the number of patent anastomoses by placental injections studies. ${ }^{6,7}$ It

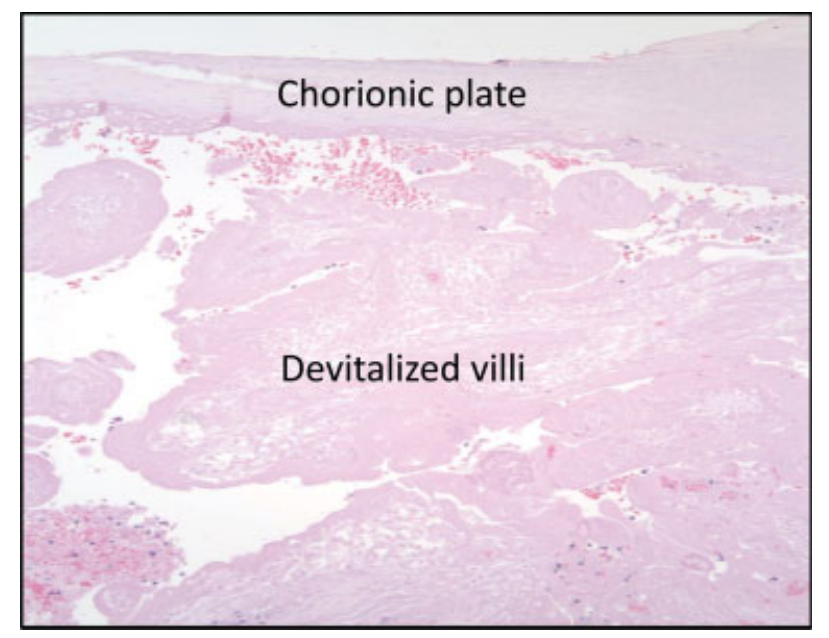

Fig. 3 H\&E-stained section $(\times 10)$ of the solomonization line showing the avascular chorionic plate and superficial underlying villi. $H \& E$, hematoxylin-eosin.

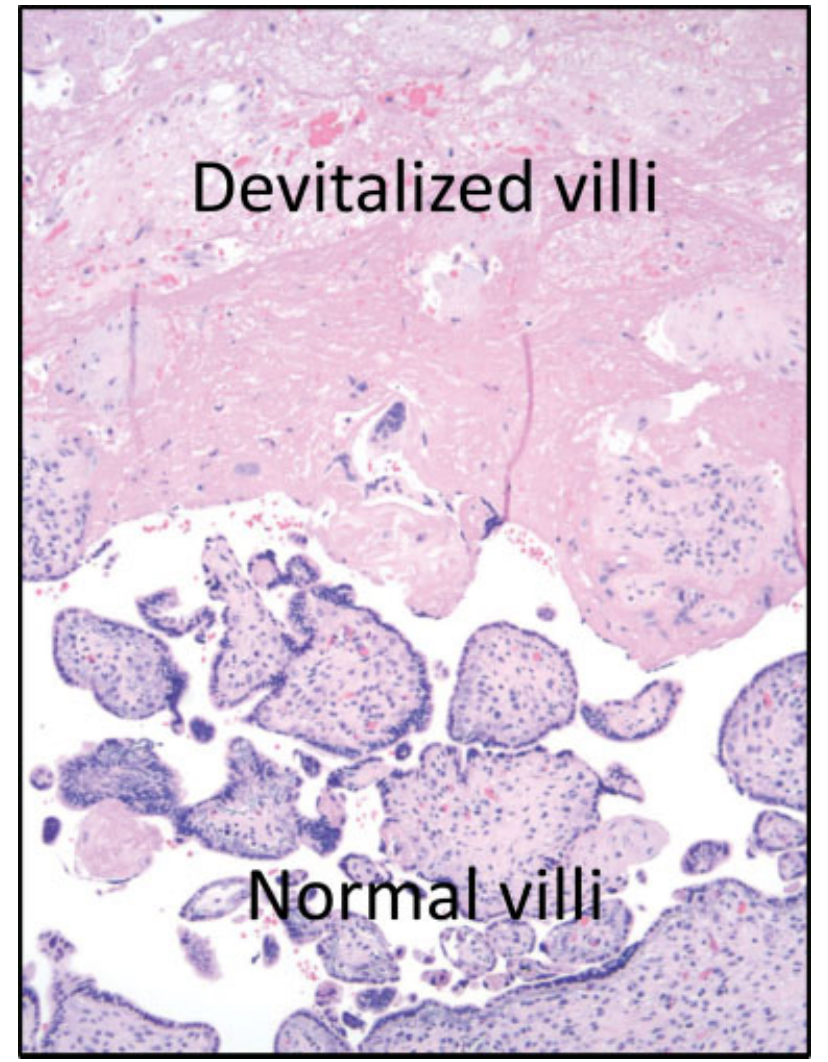

Fig. 4 H\&E-stained section $(\times 10)$ of the solomonization line showing the abrupt vascular-avascular transition within the villi adjacent to the chorionic plate. H\&E, hematoxylin-eosin.

seems reasonable that this should become the new standard when technically feasible. Questions remain, however, regarding the depth of damage to the chorionic plate and underlying villi, and potential clinical consequences such as hemorrhage, membrane rupture, or placental necrosis. In a randomized controlled trial of laser coagulation of the vascular equator versus selective coagulation, Slaghekke et al found no difference in the rate of procedure-related complications or major adverse outcomes. ${ }^{7}$ Our report has demonstrated that solomonization results in the full-thickness devitalization of the chorionic plate and only shallow devitalization of the underlying villi. Therefore, any small vessel within the line of solomonization would likely be cauterized, and the technique had little effect on the underlying placenta.

A weakness of our study is that it describes histological findings in only a single placenta. We chose to evaluate this placenta for two reasons. First, this case was technically straightforward (posterolateral placental location, clear amniotic fluid, complete visualization of the vascular equator) and allowed for complete and even application of laser energy along the Solomon line without interruption. Second, the relatively short interval between treatment and delivery allows for isolation of treatment effect before other events that might occur later in pregnancy (ongoing placental growth, hemorrhage, infarct, etc.) could distort the histology.

Using placental vascular casts, Wee et al demonstrated deep vascular anastomoses beneath the chorionic plate in 


\section{Untreated}

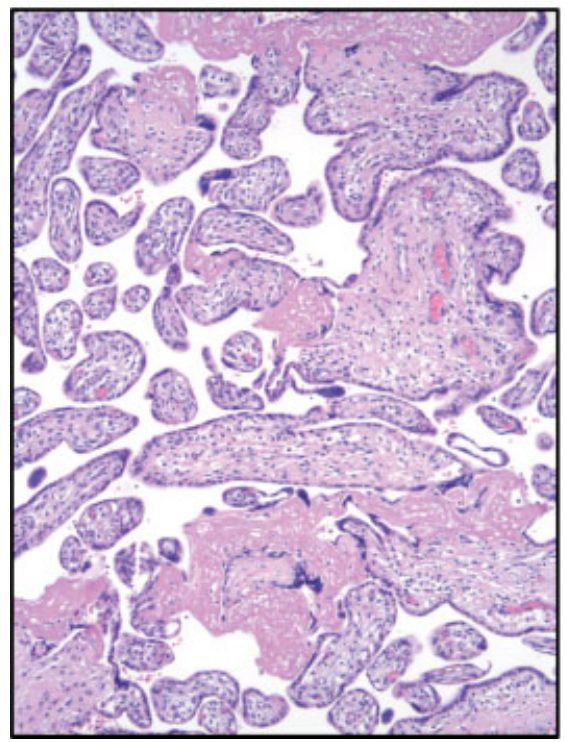

\section{Treated}

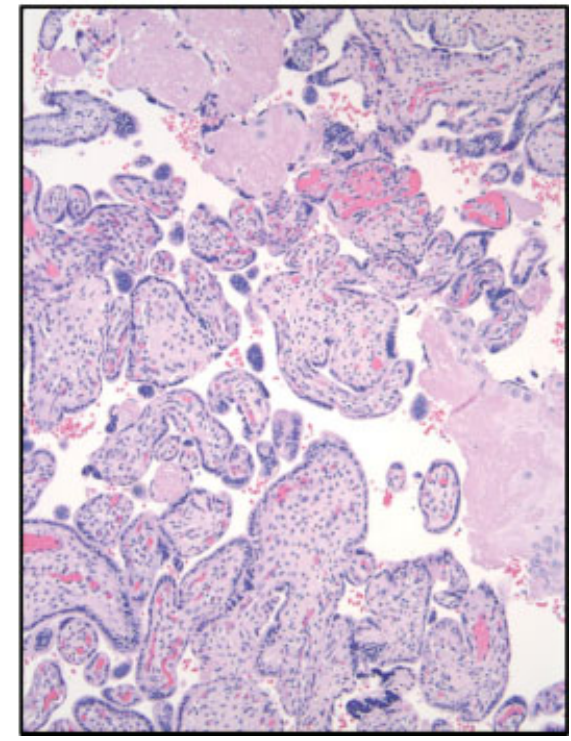

Fig. $5 \mathrm{H} \&$ E-stained sections $(\times 10)$. Compared to an untreated section from elsewhere in the placenta (left), the treated section (right) demonstrates intact villi more towards the maternal surface. H\&E, hematoxylin-eosin.

over half of monochorionic placentas. ${ }^{8}$ Lewi et al found similar deep anastomoses between cotyledons using placental angiography in placentas treated with laser coagulation. ${ }^{5}$ It would not be reasonable to assume, therefore, that solomonization will effectively treat these vessels, since the depth of devascularization was not significantly beyond the chorionic plate in the present study. For this reason, fetoscopic laser coagulation with equatorialization

Table 1 Measured depth of avascularization for each serial section along the chorionic plate of the solomonization line

\begin{tabular}{|l|l|}
\hline Section & $\mu \mathrm{m}$ \\
\hline 1E piece 1 & 4,124 \\
\hline 1E piece 2 & 3,081 \\
\hline 1F piece 1 & 1,787 \\
\hline 1F piece 2 & 2,823 \\
\hline 1G piece 1 & 3,652 \\
\hline $1 \mathrm{G}$ piece 2 & 2,431 \\
\hline $1 \mathrm{H}$ & 4,174 \\
\hline $1 \mathrm{I}$ & 2,438 \\
\hline $1 \mathrm{~J}$ & 3,852 \\
\hline $1 \mathrm{~K}$ & 2,120 \\
\hline $1 \mathrm{~L}$ & 1,269 \\
\hline $1 \mathrm{M}$ & 2,431 \\
\hline $1 \mathrm{~N}$ & 3,215 \\
\hline Range & $1,029-4,174$ \\
\hline Mean & 2,597 \\
\hline & \\
\hline
\end{tabular}

\section{Treated anastomosis}

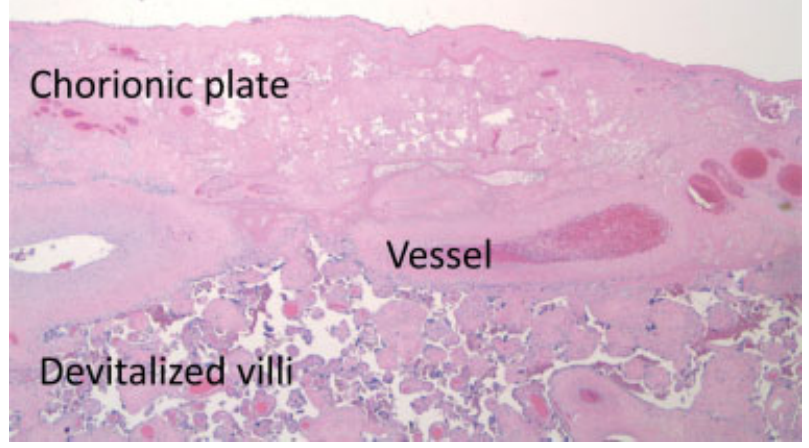

Fig. $6 \mathrm{H} \& \mathrm{E}$-stained section $(\times 4)$ of a treated anastomosis. H\&E, hematoxylin-eosin.

cannot produce complete "dichorionization," and use of the term "dichorionization" may provide a false sense of security to referring providers after laser treatment. Treated patients will still require ongoing sonographic surveillance.

Note

The University of Pittsburgh Institutional Review Board approved this study. Teaching points: (1) Placental laser equatorialization, or solomonization, produces full-thickness devitalization of the vascular equator; (2) solomonization has little effect on the underlying villi; and (3) solomonization does not produce dichorionization. 


\section{Conflict of Interest}

The authors report no conflict of interest.

\section{References}

1 Lewi L, Jani J, Blickstein I, et al. The outcome of monochorionic diamniotic twin gestations in the era of invasive fetal therapy: a prospective cohort study. Am J Obstet Gynecol 2008;199(5):514. e1-514.e8

2 Senat MV, Deprest J, Boulvain M, Paupe A, Winer N, Ville Y. Endoscopic laser surgery versus serial amnioreduction for severe twin-to-twin transfusion syndrome. N Engl J Med 2004;351(2):136-144

3 Simpson LL; Society for Maternal-Fetal Medicine. Twin-twin transfusion syndrome. Am J Obstet Gynecol 2013;208(1):3-18

4 Robyr R, Lewi L, Salomon LJ, et al. Prevalence and management of late fetal complications following successful selective laser coagu- lation of chorionic plate anastomoses in twin-to-twin transfusion syndrome. Am J Obstet Gynecol 2006;194(3):796-803

5 Lewi L, Jani J, Cannie M, et al. Intertwin anastomoses in monochorionic placentas after fetoscopic laser coagulation for twin-totwin transfusion syndrome: is there more than meets the eye? Am J Obstet Gynecol 2006;194(3):790-795

6 Slaghekke F, Lewi L, Middeldorp JM, et al. Residual anastomoses in twin-twin transfusion syndrome after laser: the Solomon randomized trial. Am J Obstet Gynecol 2014;211(3):285.e1-285.e7

7 Slaghekke F, Lopriore E, Lewi L, et al. Fetoscopic laser coagulation of the vascular equator versus selective coagulation for twin-to-twin transfusion syndrome: an open-label randomised controlled trial. Lancet 2014;383(9935):2144-2151

8 Wee LY, Taylor M, Watkins N, Franke V, Parker K, Fisk NM. Characterisation of deep arterio-venous anastomoses within monochorionic placentae by vascular casting. Placenta 2005; 26(1):19-24 